\title{
Microscopy and Microanalysis of Grain Boundaries in Cerium Oxide
}

\author{
J.P. Winterstein, ${ }^{*} \uparrow$ C.B. Carter, ${ }^{*}$ W. Grogger $\dagger$ and F. Hofer $\dagger$ \\ * Department of Chemical, Materials \& Biomolecular Engineering, University of Connecticut, \\ Storrs, CT 06269 \\ $\dagger$ Research Institute for Electron Microscopy and Center for Electron Microscopy (FELMI/ZFE), \\ Graz University of Technology, A-8010 Graz, Austria
}

Doped cerium oxide may replace zirconium oxide as the electrolyte of choice in solid-oxide fuel cells due to its higher ionic conductivity for lower temperature $\left(\sim 500^{\circ} \mathrm{C}\right)$ operation. Depending on the processing conditions (impurity level, grain size, etc.) the grain boundaries in cerium oxide can account for a large portion or the majority of the ionic resistance; with silica impurities the grain boundaries dominate the total resistance and even in high-purity samples, $\sim 1 / 3$ of the resistance has been reported to be due to the grain boundaries [1]. Grain-boundary doping with Fe has also been used to improve both the sintering and ionic conductivity of cerium oxide. Different explanations for the influence of Fe on the conductivity have been proposed including modification of the space charge and scavenging of Si impurities [2,3]. The grain boundaries in Gd-doped cerium oxide with and without $\mathrm{Fe}$, and Fe-doped cerium oxide without Gd were investigated in the TEM using both imaging and EELS.

Specimens were prepared from sintered compacts of nanoparticles produced using an aqueous solution of the desired metal nitrates and an amine [4]. The Gd concentration was $\sim 10 \mathrm{~mol} \%$ and the Fe was $\sim 0.34 \mathrm{~mol} \%$. After sintering, $3 \mathrm{~mm}$ disks were ultrasonically cut and specimens thinned using dimpling and ion milling. Microscopy and EELS were performed using a FEI Tecnai F20 with a Gatan image filter; observations made with and without a monochromator will be compared.

No Fe was detected within the grains of the cerium oxide, but STEM-EELS line profiles revealed the presence of a small amount of $\mathrm{Fe}$ at the grain boundary. Occasionally iron oxide precipitates were found in the specimen. These were always connected to a grain boundary and never observed isolated within the bulk of a cerium oxide grain. EELS and diffraction measurements indicate that the iron oxide is present as the hematite phase. Figure 1 shows two images of iron oxide particles, one at a triple junction and the other pinning a grain boundary.

STEM-EELS also reveals changes in the $\mathrm{O} \mathrm{K}$ edge and $\mathrm{Ce} \mathrm{M}$ edge at the grain boundaries. At the grain boundary, some $\mathrm{Ce}^{4+}$ ions are reduced to $\mathrm{Ce}^{3+}$ as revealed by changes in the $\mathrm{Ce}_{4,5}$ edge. Changes in the $\mathrm{O} \mathrm{K}$ edge fine structure show similarities to observations of reduced oxygen coordination in other oxides [5], but differ from observations on beam-damaged cerium oxide [6].

The imaging and EELS results are interpreted in terms of the role of grain boundaries in the performance of fuel-cell electrolytes [7].

References

[1] R. Gerhardt and A.S. Nowick, J Am Ceram Soc. 69 (1986) 641. 
[2] H.J. Avila-Paredes and S. Kim, Solid State Ion, 177 (2006) 3075.

[3] Zhang, et al., Solid State Ion, 167 (2004) 203.

[4] J. Basu, et al., Appl Surf Sci, in press (2010).

[5] N.D. Browning, et al, Phys Rev B, 58, no. 13 (1998) 8289.

[6] L.A.J. Garvie and P.R. Buseck, J Phys Chem Sol, 60 (1999) 1943.

[7] JPW is supported by the US and Austrian governments through a Fulbright grant.

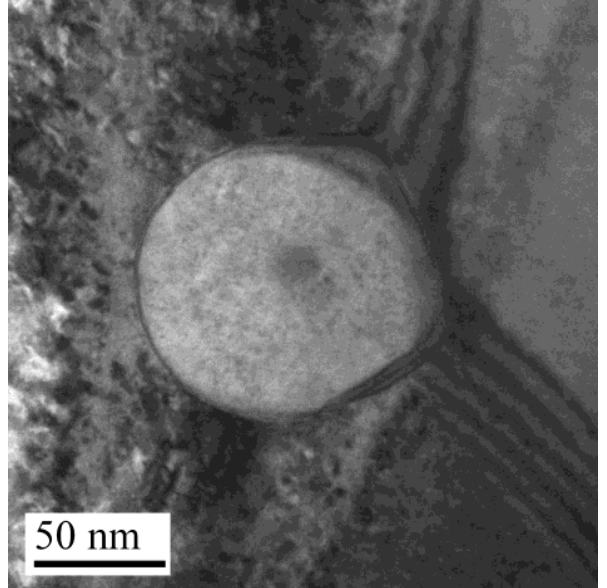

(a)

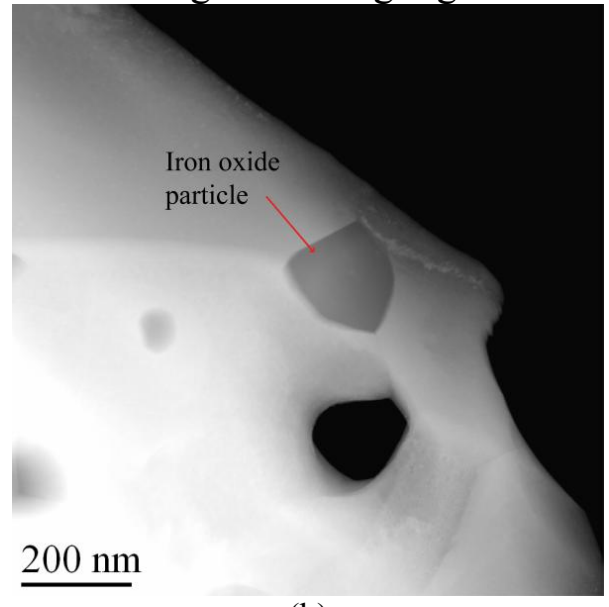

(b)

FIG 1. Polycrystalline cerium oxide: a) BF TEM image of iron oxide particle pinning a grain boundary; b) DF STEM image of an iron oxide particle at a triple junction.
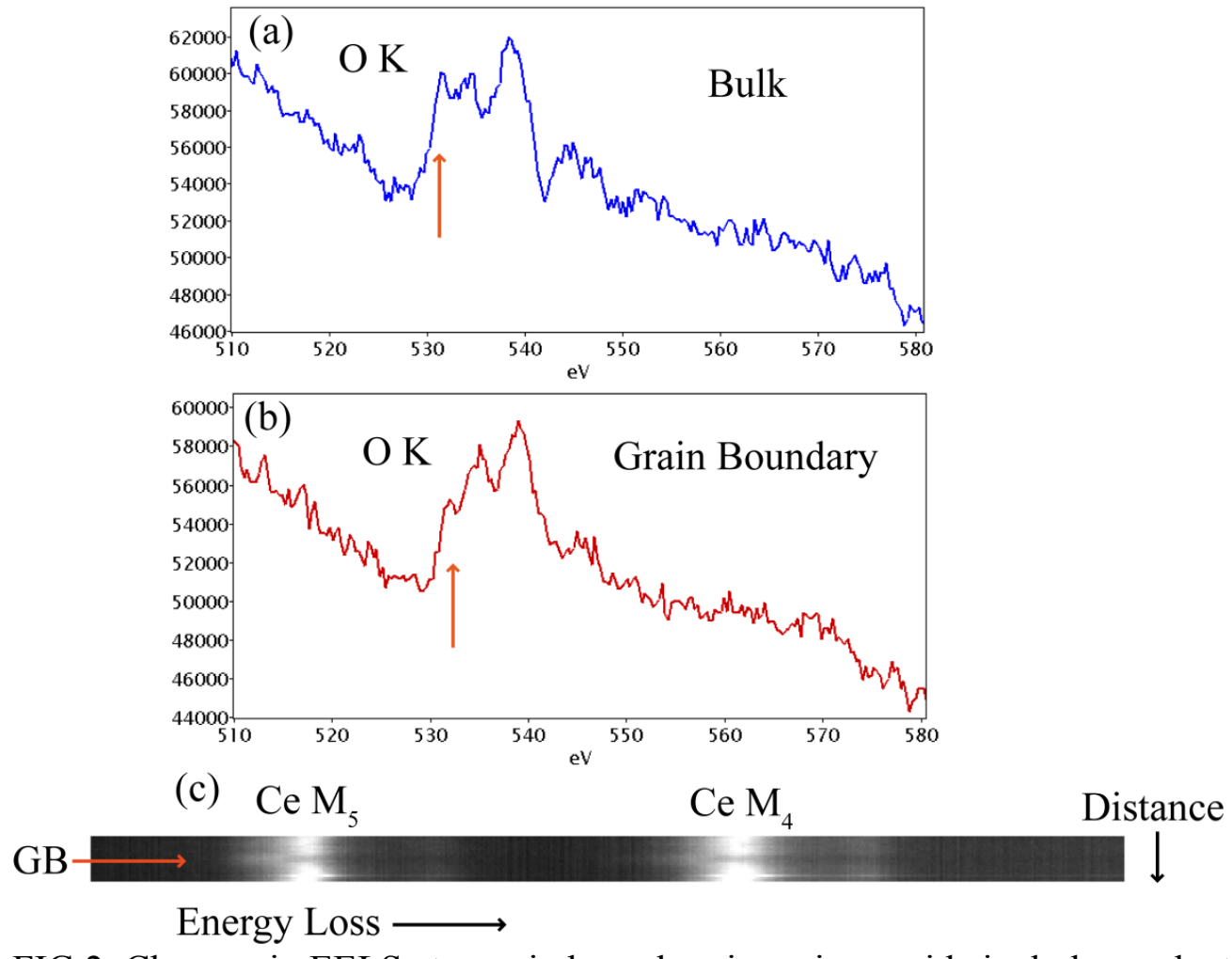

FIG 2. Changes in EELS at a grain boundary in cerium oxide include a reduction in the intensity of the first peak in the O K edge as shown by the arrows in (a) and (b); c) a shift of intensity to lower energy in the Ce $\mathrm{M}$ edge shown in the data from a STEM-EELS line scan across a grain boundary. 